\title{
Estudios de adaptación y manejo integrado de estevia (Stevia rebaudiana Bert.): nueva alternativa agroindustrial del Caribe colombiano. Una revisión
}

\section{Environmental suitability and integrated management studies in stevia (Stevia rebaudiana Bert.): a new agro-industrial alternative for the Colombian Caribbean. A review}
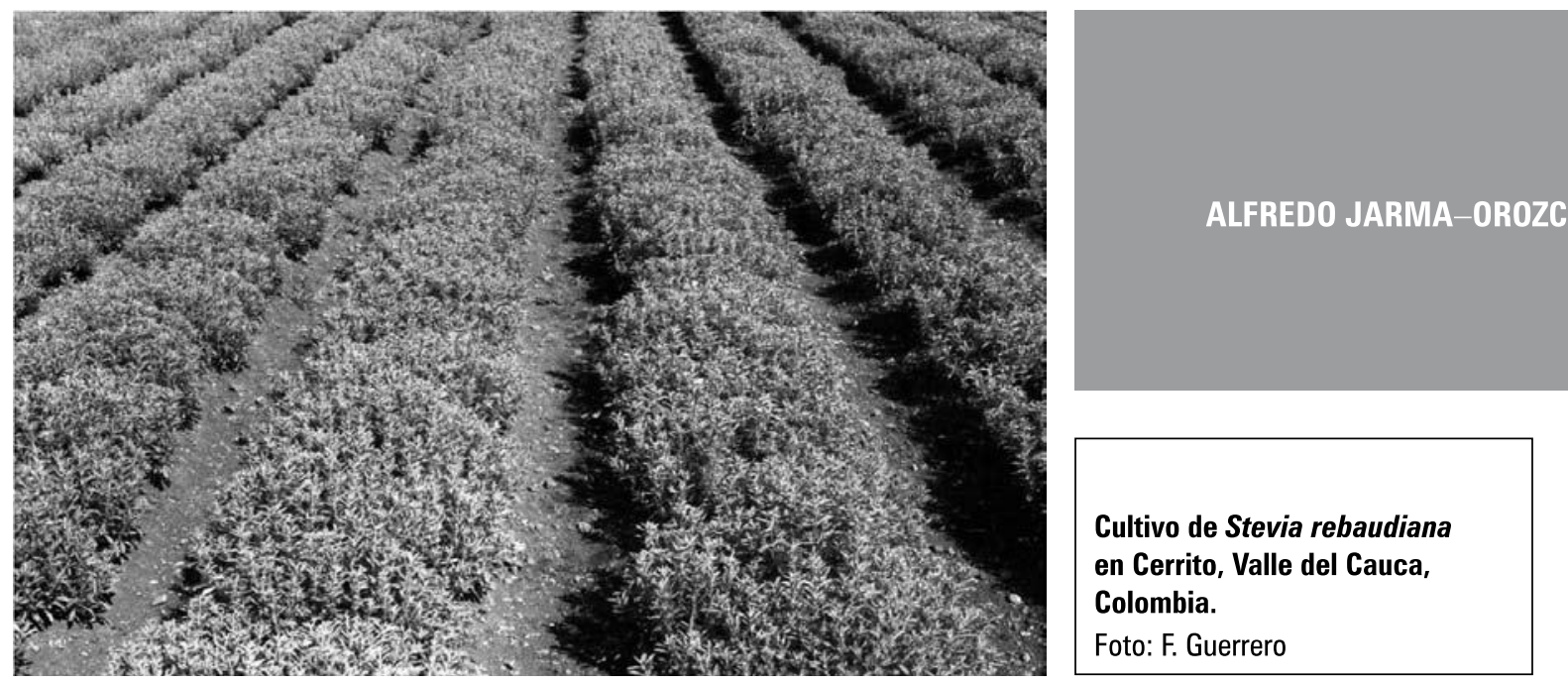

\section{RESUMEN}

En el sector rural del Caribe colombiano, la escasez de alternativas rentables de producción ha generado una reducción en el área cultivada en 380.000 ha, con la pérdida de más de cien mil empleos en la última década. Ante este escenario, la Universidad de Córdoba (Colombia) estudia la adaptación y manejo de nuevas especies agroindustriales que por su rentabilidad minimicen la migración de productores a las ciudades y contribuyan a mejorar la calidad de vida. Hace siete años se iniciaron las primeras investigaciones con estevia en aspectos como ecofisiología, nutrición, fitopatología, entre otros. Los resultados indican que estevia puede cultivarse con éxito en esta región del país. Esta planta contiene glucósidos bajos en calorías, cuyo poder edulcorante en estado puro y cristalino puede llegar a ser entre 200 y 400 veces mayor que el azúcar de caña. Adicionalmente, muchas de las labores que demanda su producción la convierten en un sistema altamente generador de empleo rural, además de los beneficios que su uso trae a la salud. El presente documento resume algunos de los resultados más importantes de las investigaciones que ha adelantado la Universidad de Córdoba con esta especie.

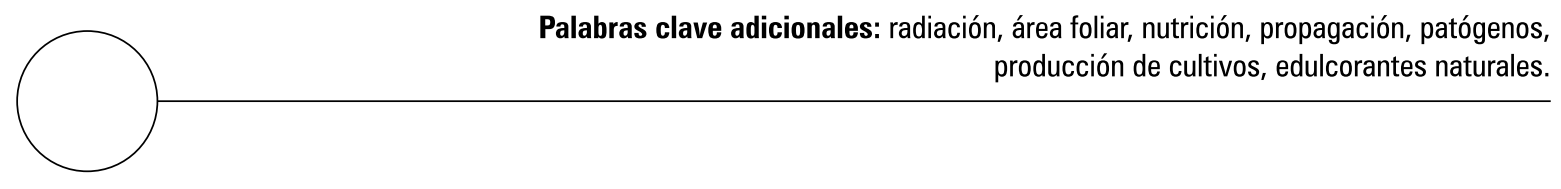

Facultad de Ciencias Agrícolas, Universidad de Córdoba, Montería (Colombia). Grupo Regional de Investigación Participativa de los Pequeños Productores de la Costa Atlántica. ajarma@sinu.unicordoba.edu.co 


\section{ABSTRACT}

Lack of profitable production alternatives in the Colombian Caribbean rural sector has generated a reduction in the cultivated area of roughly 380,000 ha, with the loss of more than 100,000 jobs in the last decade. Faced with this situation, Cordoba University (Colombia) has been studying the adaptation and integrated management of new plant species that might offer profitable alternatives to the agricultural industry and thereby minimize migration to the cities and contribute to improved quality of their life. For the last seven years, the Faculty of Agricultural Sciences has conducted studies with stevia including aspects of plant physiology, nutrition, phytopathology and propagation, among others. The results indicate that this crop can be cultivated successfully in the Colombian Caribbean. This plant contains low caloric glycosides, whose sweeting power in pure and crystal-clear state can reach values of between 200 and 400-times that of sugarcane. In addition to its health benefits for the consumer, the farm work required for stevia production is a strong generator of employment opportunities. The present document covers some of the most important results of research that has been carried out in Cordoba University with this crop.

Additional keywords: radiation, leaf area, nutrition, propagation, pathogens, crop production, natural sweeteners.

Fecha de recepción: 11-12-2007

Aprobado para publicación: 03-06-2008
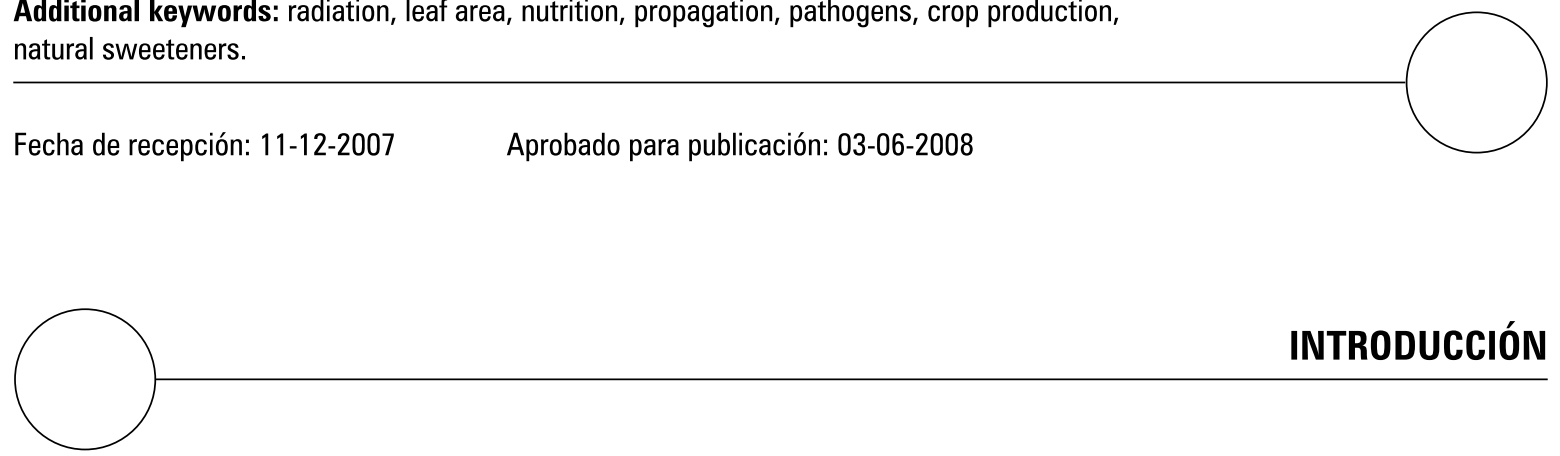

La estevia es una planta selvática subtropical del alto Paraná, nativa del noroeste de la provincia de Misiones, en el Paraguay, donde era utilizada por los nativos del lugar como edulcorante y curativa. En 1899, el sabio Moisés Santiago Bertoni obtuvo plantas por primera vez de los indígenas nativos, las cuales cultivó y finalmente en 1905 la clasificó (Ministerio Agricultura y Ganadería del Paraguay, 1996).

Los componentes edulcorantes de las hojas de estevia son glucósidos de diterpeno sintetizados, al menos en los estados iniciales, usando la misma ruta del ácido giberélico a partir del mevalonato (Kasahara et al., 2002; Hsieh y Goodman, 2005; Guevara et al., 2005), con la diferencia que en estevia, el kaureno, precursor de dichas hormonas, se convierte a esteviol en el retículo endoplásmico. Numerosos investigadores han reportado la ruta de síntesis de los edulcorantes más impor- tantes de S. rebaudiana (Totté et al., 2000; Brandle et al., 2002; Geuns, 2003; Totté et al., 2003; Brandle, 2005).

Se conoce actualmente que la estevia tiene varios componentes edulcorantes, entre los cuales la concentración en la hojas de los cuatro más importantes son: esteviósido (5-10\%), rebaudiósido A (2-4\%), rebaudiósido C (1-2\%) y dulcósido A (0,5-1\%) (Rirdc, 2005). El edulcorante es un polvo cristalino blanco que se extrae de las hojas, $100 \%$ natural, no tiene calorías y puede llegar a ser hasta 300 veces más dulce que la sacarosa y, comparativamente, es similar al aspartame (Prakash et al., 2008); las hojas pueden utilizarse en su estado natural y sólo son necesarias cantidades pequeñas del producto, gracias a su gran poder edulcorante. Puede tener efectos benéficos sobre la salud humana (Tadhani et al., 2007); puede ser utilizado por diabéticos tipo II, 
ya que no se acumula en el cuerpo y reduce los niveles de glucosa en la sangre (Jeppesen et al., 2000, 2002; Geuns et al., 2007; Brandle y Telmer, 2007), no aporta calorías al ser metabolizado, antihipertensivo (Chan et al., 2000; Lee et al., 2001; Hsieh et al., 2003; Gregersen et al., 2004), antiácido, cardiotónico, no produce caries, al no ser fermentado por las bacterias orales, presenta actividad antirotavirus (Das et al., 1992) y se distingue de los edulcorantes artificiales por no tener sabor metálico y no ser cancerígeno (Brandle, 2005; Galván et al., 2003; Jarma-Orozco et al., 2005; Jarma-Orozco et al., 2003).

Con la difusión de las informaciones sobre la naturaleza y los usos potenciales de los principios edulcorantes de esta planta, su cultivo comercial adquirió importancia variable en Brasil, Japón, Corea, Taiwán, Tailandia, Indonesia, Laos, Malasia, China, Filipinas, Estados Unidos (California) y, lógicamente, en el Paraguay (Marín, 2004). El 5\% de la producción mundial actual se concentra en el noroeste asiático (Koyama et al., 2003). Japón es el país con el mayor número de fábricas procesadoras y extractoras de esteviósido (Schwebel, 2005).

En Colombia se tienen informes sobre la introducción de estevia al Valle del Cauca y Antioquia iniciando la década del noventa, desconociéndose su procedencia y año de introducción. En la actualidad hay registros de la siembra de esta especie en Antioquia, Córdoba, Tolima, Huila, Valle del Cauca y Meta.

En el cultivo de estevia se utilizan cerca de 245 jornales permanentes por ha y año, por lo que puede plantearse como alternativa para generación de empleo, además de contribuir a la diversificación de los renglones agrícolas de la región y el país (Secretaría de Agricultura de Antioquia, 2000). Sin embargo, por ser un cultivo de reciente introducción, las técnicas de manejo, los genotipos utilizados, los requerimientos hídricos y nutricionales $y$, en general, el desconocimiento del mismo, han sido limitantes para una producción sostenible y eficiente (Eafit, 2004).
El éxito de cualquier cultivo depende en principio de los conocimientos que sobre éste se tengan. Es clave entender la fisiología de la especie en función del ambiente para implementar las prácticas de cultivo que optimicen la producción. Diversos factores como la oferta de la radiación, el tipo de suelos, la nutrición, la densidad de poblaciones, etc. desempeñan un papel fundamental y determinante, porque a partir de su interacción se sintetizarán gran cantidad de compuestos metabólicos.

En Colombia, la Universidad de Córdoba ha venido desarrollando durante siete años trabajos de investigación con esta especie, que apuntan a definir paquetes tecnológicos de manejo agronómico (requerimientos nutricionales, respuesta a la radiación, tipos de suelo, entre otros) y cuyos resultados muestran que es viable la posibilidad de cultivarse en la costa caribeña colombiana. Sin embargo, aún faltan estudios científicos que indiquen cómo crece y se desarrolla la especie ante diversos factores y la manera en que éstos afectan la calidad de los edulcorantes (JarmaOrozco, 2006). Por esta razón, este documento presenta una relación de los principales avances en la investigación que ha adelantado la Universidad de Córdoba en estevia con miras de determinar la factibilidad agronómica del cultivo en la región del Caribe húmedo de Colombia y definir las prioridades de investigación.

\section{RADIACIÓN}

Con el propósito de evaluar el efecto de cuatro niveles de radiación incidente (RI) sobre el comportamiento fisiológico de estevia, se realizó una investigación entre julio de 2002 y abril de 2003 en la Facultad de Ciencias Agrícolas de la Universidad de Córdoba (Montería). Para lograrlo, se planteó un diseño completo al azar con arreglo factorial de $4 \times 2$, en el que el factor A correspondió a los niveles de RI (19, 24, 56 y 100\%) y el factor $\mathrm{B}$ a los genotipos conocidos en Colombia como 'Morita 1' y 'Morita 2'. Los mayores registros de RI se dieron a las 11:00 horas, observán- 
dose valores de $3.885,2.132,891,5$ y $741,3 \mu \mathrm{mol}$ $\mathrm{m}^{-2} \mathrm{~s}^{-1}$ para los niveles $100,56,24$ y $19 \%$, respectivamente (Rengifo et al., 2003). Los principales resultados indicaron que el área foliar por planta de 'Morita 2' fue significativamente superior al de 'Morita 1' $(P \leq 0,05)$, independientemente del nivel de radiación (tabla 1) e igualmente los altos niveles de RI (100 y 56\%) registraron la mayor acumulación de biomasa de las hojas, siendo superior en 'Morita 2' durante todo el ciclo de evaluación (figura 1). Por su parte, la interacción que evaluó la tasa absoluta de crecimiento (TAC) indicó que de manera consistente con lo anterior, 'Morita 2' expuesta al 100\% de radiación incidente presentó valores superiores de este índice respecto a 'Morita 1'.
Los resultados indicarían que estevia se comportaría como especie heliófila para estas condiciones de Colombia, aunque sería importante encontrar los puntos de saturación lumínica, al considerar que en la región se presentan radiaciones muy altas (como las registradas a un nivel de $100 \%$ ), las cuales pueden causar daños al sistema oxidativo de la planta, aparentemente de manera más importante en 'Morita 1' respecto a 'Morita 2'. Algunos autores han indicado en otras especies que los factores de cultivo pueden afectar diferencialmente la expresión del área foliar y la asimilación de biomasa en función de la radiación interceptada y de la especie (Lizaso et al., 2003; Mansab et al., 2003; Blanco y Folegatti 2005; Tewolde et al., 2005;). El comportamiento

Tabla 1. Área foliar total por planta $\left(\mathrm{cm}^{2}\right)$ de dos genotipos de estevia en el Valle del Sinú, Colombia.

\begin{tabular}{|l|c|c|c|}
\multirow{2}{*}{ Genotipo } & \multicolumn{3}{|c|}{ Días después del trasplante } \\
\cline { 2 - 4 } & 60 & 150 & 225 \\
\hline 'Morita 1' & $71 \mathrm{a}^{*}$ & $239 \mathrm{a}$ & $878 \mathrm{a}$ \\
\hline 'Morita 2' & $186 \mathrm{~b}$ & $399 \mathrm{~b}$ & $1700 \mathrm{~b}$ \\
\hline
\end{tabular}

*: Promedios con letras distintas en una misma columna indican diferencia significativa de acuerdo al test protegido de DMS $(P \leq 0,05)$.

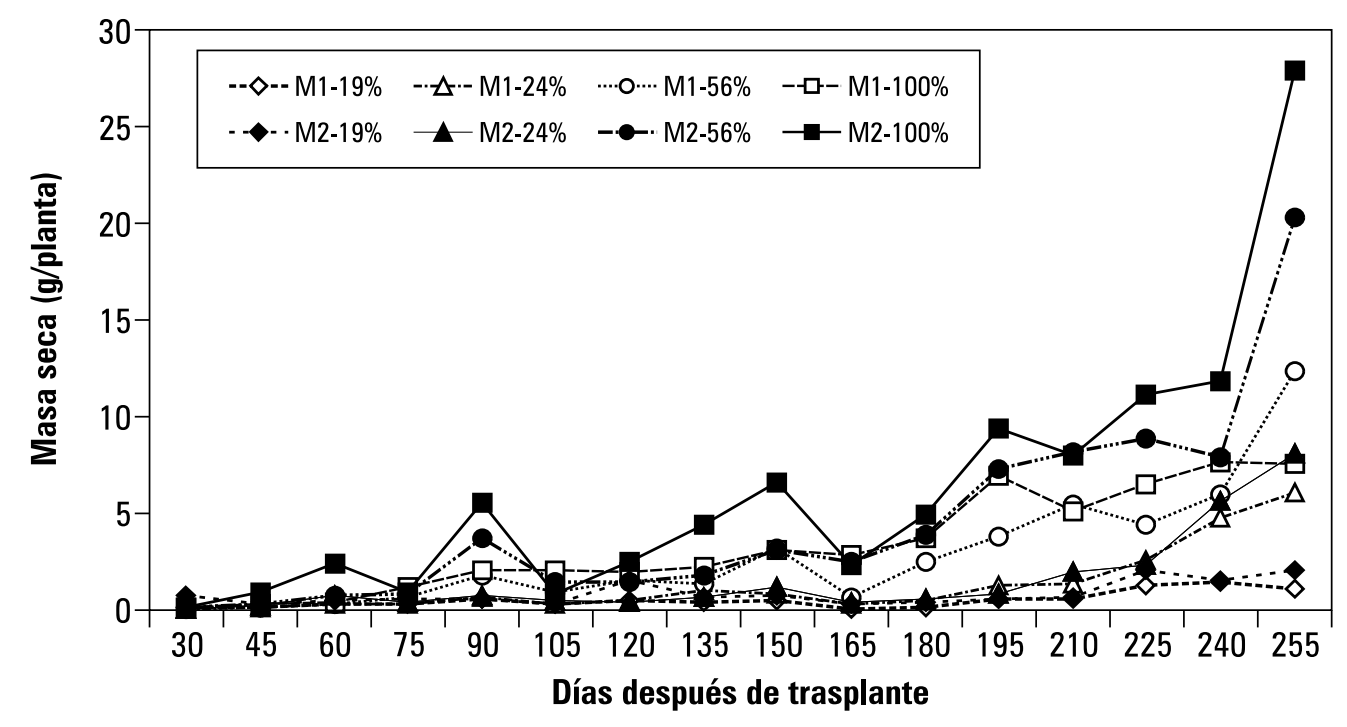

Figura 1. Masa seca de hojas por planta de dos genotipos de estevia (M1 = Morita 1; $M 2=$ Morita 2) bajo cuatro niveles de radiación incidente (\%) en el Valle del Sinú del Caribe colombiano, según Jarma-Orozco et al. (2005). 
diferencial entre los genotipos evaluados supone que las divergencias genéticas favorecen a 'Morita 2' respecto a su eficiencia en la síntesis de asimilados bajo condiciones de alta radiación, que son propias de esta región del país (JarmaOrozco et al., 2005, 2006).

\section{NUTRICIÓN}

En una investigación desarrollada en 2002, se cuantificó el efecto de la aplicación de dosis combinadas de los elementos N, Py K sobre variables morfológicas y fisiológicas de 'Morita 2' y se determinaron los contenidos de estos elementos en la planta en diferentes estados de desarrollo. Se utilizó un diseño completamente aleatorio con tres repeticiones y los análisis se hicieron bajo la metodología de superficie respuesta (Galván et al., 2003).

Los principales hallazgos indican que, para la producción de 2,5 t ha ${ }^{-1}$ de hoja seca, las plantas cultivadas bajo la oferta ambiental de Córdoba requieren aproximadamente la mitad de $\mathrm{N}$ y $\mathrm{P}$ y la cuarta parte de $\mathrm{K}$ a los 60 días después del trasplante (tabla 2), en comparación con algunos reportes de investigadores de Brasil, que indican que a esta edad el cultivo requiere 325,47 y 330 $\mathrm{kg} \mathrm{ha}^{-1}$ de N, P y K, respectivamente (De Lima y Malavolta, 1997; De Lima et al., 1997 a y 1997 b). Las diferencias podrían obedecer a que a los 60 ddt la edad fisiológica de las plantas fue diferencial, adicionalmente a que los requerimientos nutricionales varían con la oferta ambiental de cada región, incluyendo los tipos de suelos (Peng et al., 2002; Vergara et al., 2003; Yan et al., 2006). En 2002, Marschner indicó que los estudios de nutrición deben estar dirigidos al establecimiento de la relación entre el suministro de nutrientes y el crecimiento de las plantas en términos de producción de materia seca.

Por otra parte, las respuestas observadas en la acumulación de biomasa de las hojas por planta (figura 2) mostraron que, ante la ausencia de $\mathrm{P}$, los niveles altos de $\mathrm{K}$ pueden incidir de manera negativa sobre esta biomasa, pero que a niveles medios y altos de $\mathrm{P}$ el aumento del peso seco de las hojas es directamente proporcional al incremento en los niveles de $\mathrm{K}$, lo que sin duda sugiere la importancia de mantener niveles adecuados de los dos elementos en el plan de nutrición. Asimismo, se pueden analizar los resultados en el sentido que no hay respuesta importante al $\mathrm{P}$ cuando la aplicación de $\mathrm{K}$ es cero, pero es alta y positiva cuando los niveles de $\mathrm{K}$ disponible son altos.

\section{ÁREA FOLIAR}

Con el propósito de ajustar un modelo estadístico para estimar el área foliar $(A f)$ sin utilizar muestreos destructivos Espitia et al. (2006) evaluaron los genotipos 'Morita 1' y 'Morita 2' bajo condiciones de campo; en cada uno se tomaron 20 ramas de $25 \mathrm{~cm}$ de largo distribuidas aleatoriamente dentro del dosel. Cada rama se dividió en

Tabla 2. Contenido nutricional de estevia (60 ddt) en Montería (Córdoba, Colombia) y requerimientos nutricionales de $N, P$ y $K$.

\begin{tabular}{|c|c|c|c|c|}
\hline \multirow[b]{2}{*}{ Elemento } & \multirow{2}{*}{$\begin{array}{l}\text { Contenido } \\
\text { nutricional } \\
\text { (mg/planta) }\end{array}$} & \multicolumn{3}{|c|}{ Requerimientos nutricionales $\left(\mathrm{kg} \mathrm{ha}^{-1}\right)$} \\
\hline & & $\begin{array}{l}\text { Población: } 100 \text { mil } \\
\text { plantas/ha }\end{array}$ & $\begin{array}{c}\text { Para } 1,0 \mathrm{t} \\
\text { hojas secas }\end{array}$ & Para 2,5 t hojas secas \\
\hline $\mathrm{N}$ & 149,0 & 15 & 70,0 & 175 \\
\hline $\mathrm{P}$ & 19,4 & 2 & 9,2 & 23 \\
\hline K & 71,2 & 7 & 32,0 & 80 \\
\hline
\end{tabular}




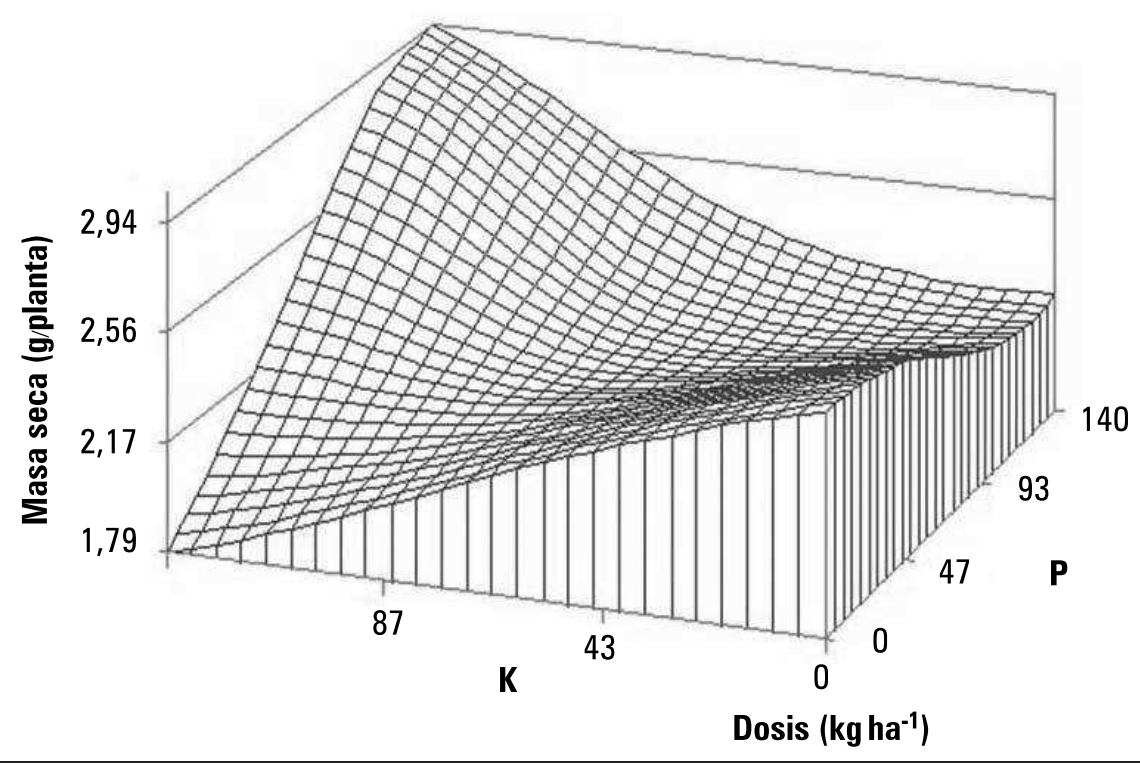

Figura 2. Masa seca de hojas en estevia ('Morita 2'), en función de dosis combinadas de P y K en el Valle del Sinú del Caribe Colombiano, según Galván et al. (2003).

tres tercios: inferior, medio y superior. De cada tercio se tomaron cinco muestras (repeticiones) de 20 hojas cada una. A cada hoja se le midió el largo y ancho máximo. Con los datos de largo del tercio inferior (LTI), medio (LTM), superior (LTS), el ancho del tercio inferior (ATI), medio (ATM) y superior (ATS) de las hojas y utilizando el método gravimétrico, se realizó un análisis de regresión lineal múltiple para definir el modelo de predicción del $A f$.

Los resultados demostraron que es posible utilizar el largo y ancho máximo de la hoja para estimar el Af. Los modelos resultantes fueron: $\sqrt{ }$ Af $=0,288+0,093 \mathrm{LTI}+0,525 \mathrm{ATI}+0,171 \mathrm{LTS}$ $\left(R^{2}=0,9604\right)$ para 'Morita 1' y $\sqrt{ } \mathrm{Af}=0,319+$ 0,223 ATI + 0,332ATM + 0,067LTS + 0,257ATS $\left(R^{2}=0,9213\right)$ para 'Morita 2'.

\section{PROPAGACIÓN}

La variabilidad genética de estevia en Colombia es limitada si se considera que en el país sólo se cultivan los genotipos 'Morita 1'y 'Morita 2'; por ello, es importante evaluar técnicas biotecnológicas para desarrollar protocolos de regeneración de plantas que puedan constituirse en soluciones para la generación de variabilidad genética en esta especie. En un trabajo adelantado por Suárez y Salgado (2008), se trataron secciones de entrenudos de plantas de estevia establecidas en condiciones in vitro con diferentes concentraciones de bencilaminopurina (BAP) combinadas con varias cantidades de ácido naftalenacético (ANA), con el propósito de evaluar su efecto sobre el porcentaje de inducción de callo, friabilidad y formación de órganos. Posteriormente, los callos inducidos fueron multiplicados y se observó el efecto de cuatro combinaciones de ANA y BAP sobre el incremento de masa fresca. Finalmente, los tallos proliferados fueron transferidos sobre medio de regeneración suplementado con diferentes cantidades de BAP o en combinación con ANA con el fin de evaluar la regeneración de brotes. Todos los tratamientos fueron distribuidos utilizando un diseño completamente aleatorio con un mínimo de 15 repeticiones por 
tratamiento. Los resultados permitieron observar que la presencia de auxina en el medio de cultivo es necesaria y suficiente para inducir la formación de callos en los explantes de estevia, por cuanto al menos el $80 \%$ desarrolló callos en presencia de ANA o ANA + BAP, lo cual concuerda con los resultados de otros investigadores cuando evaluaron el efecto de las auxinas en presencia de BAP en la formación de tejido calloso en otras especies (Solange et al., 2002; Sánchez et al., 2005). Por otro lado, menos de un $5 \%$ de los explantes cultivados en la dosis máxima de BAP (4 $\mathrm{mg} \mathrm{dm}^{-3}$ ) desarrollaron algún tipo de cultivo (figura 3).

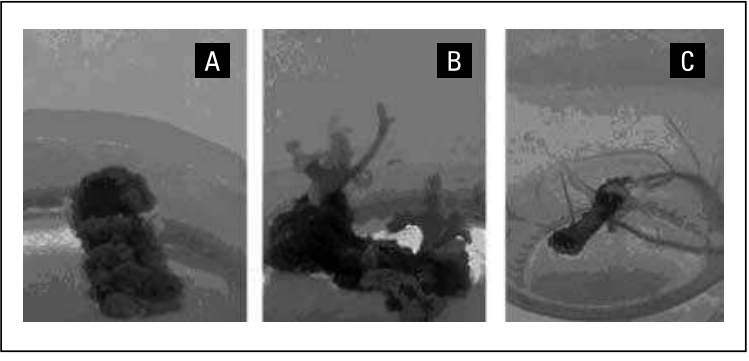

Figura 3. Organogénesis de estevia; callo (izquierda), regeneración de tallos (centro), regeneración de raíces (derecha), según Suárez y Salgado (2008).

En cuanto a la regeneración de brotes vía organogénesis, ocurrió de forma independiente a la presencia de reguladores de crecimiento en el medio, y estos tampoco afectaron el incremento de masa fresca de callo ni tuvieron efecto sobre la regeneración de plantas a partir del callo proliferado. Los resultados permiten recomendar un suplemento combinado de $1,0 \mathrm{mg} \mathrm{dm}^{-3} \mathrm{ANA}+$ 4,0 $\mathrm{mg} \mathrm{dm}^{-3}$ BAP para obtener plantas de estevia mediante organogénesis.

En otros trabajos de propagación adelantados por Caraballo (2006), tendientes a multiplicar la especie por vía sexual a través del rompimiento de latencia de las semillas, se evaluó el efecto de diferentes tipos de luz, temperaturas, hormonas $y$ sus combinaciones. Los resultados indicaron que ninguno de los factores estudiados presentó efectos positivos significativos sobre la germinación de las semillas. El autor concluyó que estos resultados pudieron obedecer al bajo porcentaje de viabilidad que presenta la semilla sexual de esta especie, cuyos porcentajes de germinación varían entre el $10 \%$ y $38 \%$ y a que la longevidad de los aquenios es corta (a los cuatro meses, el porcentaje de germinación se reduce entre un $40 \%$ y $70 \%$, después de ocho meses es casi nulo). Adicionalmente a lo anterior, es probable que estos resultados obedezcan también a que la planta presenta semillas estériles con apariencia de semillas normales, lo cual reduce el porcentaje de germinación de la misma (Felippe et al., 1971; De Vargas, 1980; Sagakuchi y Tatsuiko, 1982; Jordan, 1984; Caraballo, 2006).

Por otra parte, los estudios de propagación asexual con inducción hormonal por ácido indolbutírico (AIB) mostraron que el área foliar, la longitud de raíces y la masa seca de las hojas de 'Morita 2' fueron superiores a los de 'Morita 1', independientemente de las concentraciones de AIB aplicadas. Sin embargo, 'Morita 1' registró mayor longitud de raíces, lo cual probablemente estuvo relacionado con los contenidos endógenos de auxinas de la variedad. Las concentraciones de AIB no afectaron la tasa absoluta de crecimiento (TAC) en ninguno de los dos genotipos (JarmaOrozco, 2005).

\section{POBLACIONES}

Para determinar el efecto de diversas poblaciones por hectárea sobre los rendimientos de biomasa del cultivo con base en la oferta ambiental de Córdoba, se han adelantado trabajos con material vegetativo de estevia entre 2005 y 2007 con $62.500,125.000$ y 250.000 plantas/ha para 'Morita 1' y 'Morita 2' (Barbosa y Janna, 2007). Los resultados preliminares de esta investigación permiten inferir que, para las condiciones del Caribe colombiano en Córdoba, el comportamiento de 'Morita 1' y 'Morita 2' es diferente. Así, se pudo 
determinar que 'Morita 1' presenta mejores respuestas a poblaciones altas (250.000 plantas/ha) en tanto que 'Morita 2' mantiene los rendimientos de hoja seca por hectárea en poblaciones intermedias y altas (figura 4).

\section{HONGOS FITOPATÓGENOS}

Muestreos realizados por el Laboratorio de Fitopatología de la Universidad de Córdoba, Montería, en los cultivos de estevia, han permitido determinar un complejo de hongos fitopatógenos que pueden ser limitantes de la producción si no se da un manejo adecuado de los mismos (Jarma-Orozco et al., 2003). En dicho estudio se aislaron patógenos pertenecientes a los géneros Fusarium, Sclerotium, Alternaria, Botriodiplodia, Colletotrichum, Cladosporium, Cercospora, Choanepho$\mathrm{ra}$, Corinespora y Curvularia; estos tres últimos no presentan reportes de incidencia en estevia en el país (figura 5).
La determinación de estos patógenos muestra el riesgo potencial: eventualmente pueden presentarse de forma epidémica en el cultivo, estimulados por las condiciones de alta humedad ambiental, propias de la región, y por grandes extensiones del mismo; por ello, es de importancia disponer de una mayor oferta de materiales genéticos para un futuro.

\section{USOS}

Adicionalmente a los trabajos anteriores, el grupo de investigadores de la Universidad de Córdoba ha estudiado algunos aspectos como el uso de los edulcorantes de estevia en mermeladas y néctares de guayaba (De Paula et al., 2003). Los resultados más importantes de este trabajo indicaron que es posible obtener productos vegetales como néctar y mermelada de guayaba dulce edulcorados con estevia, en los cuales se sustituye un porcentaje de sacarosa con adecuadas

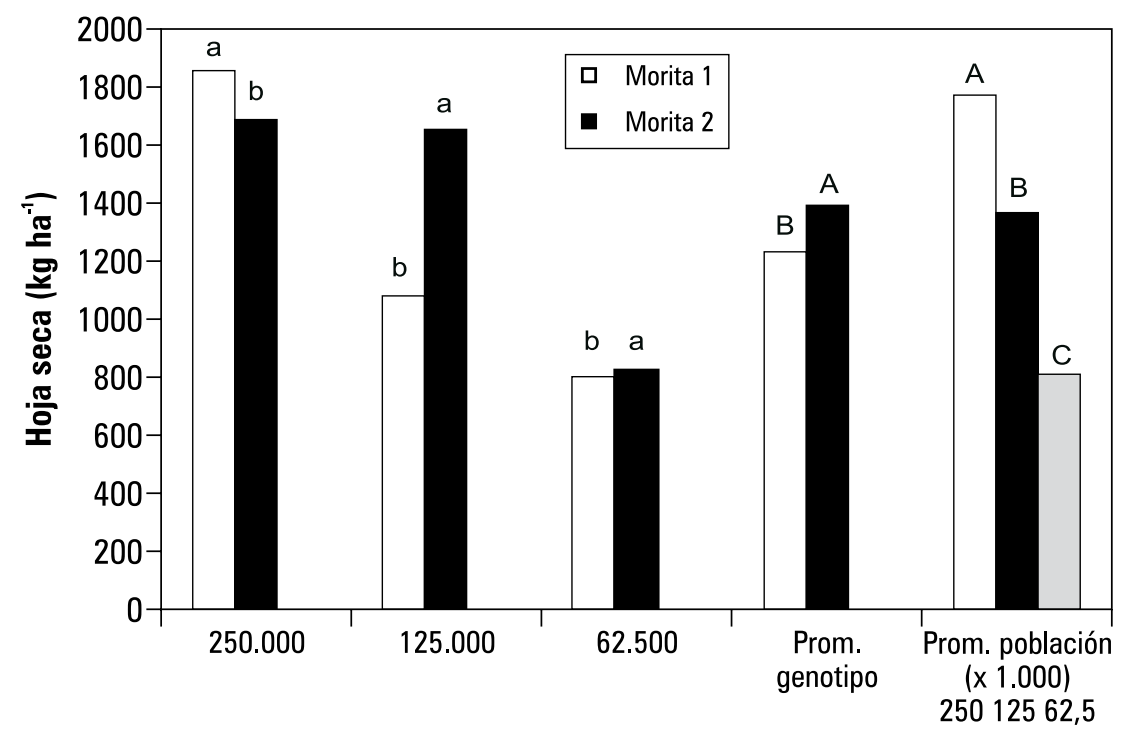

Plantas/ha

Figura 4. Rendimiento de hoja seca de dos genotipos de estevia ante tres densidades de población de plantas. 


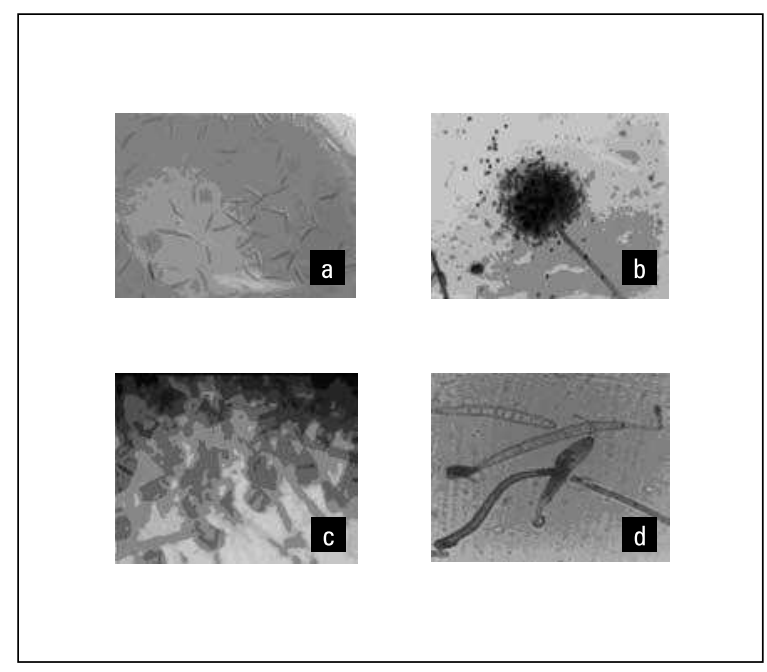

Figura 5. Algunas estructuras de géneros de hongos fitopatógenos aislados en el Laboratorio de Fitopatología de la Universidad de Córdoba a partir de hojas y tallos de estevia. a) Fusarium, b) Choanesphora, c) Curvularia y d) Corynespora, según Espitia et al. (2008).

características fisicoquímicas, microbiológicas y sensoriales. Sin embargo, sus grados Brix no serán suficientes para mantener el néctar estable por un periodo largo de tiempo, debido a que la estabilidad y conservación del mismo depende, en gran proporción, de su contenido de sólidos solubles y estos fueron bajos en los productos experimentales. Por otra parte, los investigadores informaron que con la utilización de estevia se podrán reemplazar los edulcorantes artificiales como sacarina y aspartame, empleados comúnmente para endulzar alimentos procesados, logrando con ello incursionar en el mercado con un producto que cumple con la misma función de forma natural. Ambos productos, mermelada y néctar de guayaba dulce, fueron satisfactoriamente aceptados por los consumidores.

\section{CONCLUSIONES}

Córdoba ofrece condiciones ambientales que le permiten potencializarse como un gran productor de estevia en Colombia. Factores como la oferta de luz y suelos aptos pueden convertirse en puntos estratégicos a favor de su explotación. Sin embargo, la escasa oferta de variabilidad genéti$\mathrm{ca}$, las altas humedades relativas en determinadas épocas del año y su efecto sobre la aparición de enfermedades pueden ser limitantes serias.

Las investigaciones adelantadas por la Universidad de Córdoba, aunque no todas relacionadas en este documento, pueden considerarse como un primer paso en la identificación de tecnologías competitivas, que hagan de la agroindustria de la estevia un negocio rentable y a su vez genere bienestar social en la región.

Por último, es importante recordar que el éxito de cualquier cultivo depende en gran parte de la oferta ambiental, en la que se establezcan el genotipo y las tecnologías para su producción. Una combinación adecuada de estos factores se logra cuando se tienen conocimientos objetivos de la especie, los cuales deben estar basados en resultados de investigaciones científicas que posteriormente puedan escalarse en el sistema de producción. Aunque aún falta mucho por investigar en el manejo de esta nueva especie en el país, los avances en este proceso de la Universidad de Córdoba pueden ser una motivación para animar a otras entidades que también realizan investigaciones en el sector agroindustrial de Colombia a reunir esfuerzos y recursos para trabajar en equipo en pro de una producción e industrialización sostenible de la estevia en el país. 


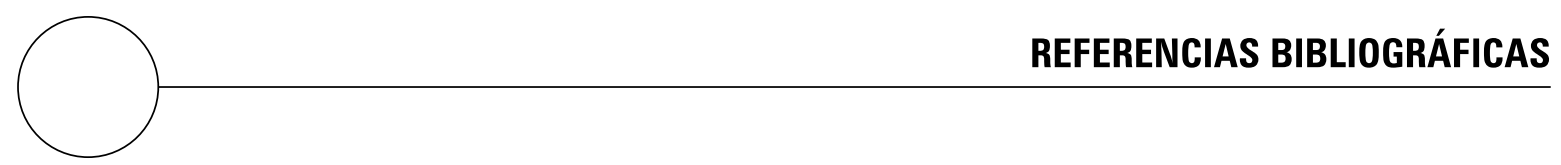

Barbosa, C. y J. Janna. 2007. Comportamiento del crecimiento de dos clones de Stevia rebaudiana Bert. bajo tres arreglos poblacionales en las condiciones ambientales del Sinú Medio. Trabajo de grado. Facultad de Ciencias Agrícolas, Universidad de Córdoba, Montería, Colombia.

Blanco, F. y M. Folegatti. 2005. Estimation of leaf area for greenhouse cucumber by linear measurements under salinity and grafting. Sci. Agric. 62, 305-309.

Brandle, J. 2005. Stevia, nature's natural low calorie sweetener. En: http://www.certifiedorganicfood stevia FAQ; consulta: junio de 2006.

Brandle, J.; A. Richman; A. Swanson y B. Chapman. 2002. Leaf ESTs from Stevia rebaudiana: a resource for gene discovery in diterpene synthesis. Plant Mol. Biol. 50, 613-622.

Brandle, J.E. y P.G. Telmer. 2007. Steviol glycoside biosynthesis. Phytochem. 68, 1855-1863.

Caraballo, U. 2006. Manejo de propágalos de Stevia rebaudiana (Bertoni) para optimizar su establecimiento bajo condiciones del Caribe Colombiano. Tesis de maestría. Facultad de Ciencias Agrícolas, Universidad de Córdoba, Montería, Colombia.

Chan, P.; B. Tomlinson; Y. Chen; J. Liu; M. Hsieh y J. Cheng. 2000. A double blind placebo-controlled study of the effectiveness and tolerability of oral stevioside in human hypertension. Brit. J. Clin. Pharmacol. 50, 215-220.

Das, S.; A. Das; R. Murphy; I. Punwani; M. Nasution y A. Kinghorn. 1992. Evaluation of the cariogenic potential of the intense natural sweeteners stevioside and rebaudioside A. Caries Res. 26, 363-366.

De Lima, O. y E. Malavolta. 1997. Sintomas de desordens nutricionais em estévia Stevia Rebaudiana (Bert.) Bertoni. Sci. Agric. 54(1-2), 53-61.

De Lima, O.; E. Malavolta; J. De Sena y J. Carneiro. 1997a. Absorção e acumulação de nutrientes em estévia Stevia rebaudiana (Bert.) Bertoni: I. Macronutrientes. Sci. Agric. 54(1-2), 23-30.

De Lima, O.; E. Malavolta; J. De Sena y J. Carneiro 1997b. Absorção e acumulação de nutrientes em Estévia Stevia rebaudiana (Bert.) Bertoni: II. Micronutrientes. Sci. Agric. 54(1-2), 14-22.
De Paula, C.; A. Carmona y G. Lombana. 2003. Determinación de las condiciones de utilización del edulcorante natural Stevia rebaudiana Bert., en el proceso de elaboración de derivados vegetales (mermelada y néctar de guayaba dulce). Trabajo de grado. Escuela de Ingeniería de Alimentos, Universidad de Córdoba, Montería, Colombia.

De Vargas, R. 1980. Informe sobre viaje al Japón para observar la producción, comercialización e industrialización de la planta Stevia rebaudiana Bertoni. Asunción, Paraguay.

Eafit, Escuela de Administración y Finanzas. 2004. Inteligencia de mercados internacionales de $S$. rebaudiana. Departamento de Negocios Internacionales, Medellín, Colombia.

Espitia, M.; R. Montoya y A. Jarma. 2008. Stevia en el Caribe Colombiano. Facultad de Ciencias Agrícolas, Universidad de Córdoba, Montería, Colombia.

Espitia, M.; R. Montoya; J. Robles; C. Barbosa y C. Vergara, C. 2006. Modelo estadístico para estimación del área foliar en Stevia rebaudiana Bertoni en el Sinú Medio. Temas Agrarios 11(2), 45-51.

Felippe, G.; N. Lucas; L. Behar y M. Oliveira. 1971. Observaçoes a respeito da germinaçao de Stevia rebaudiana Bert. Hoehnea 1, 81- 91.

Galván, L.; J. Guzmán; A. Jarma y E. Combatt. 2003. Determinación de los requerimientos nutricionales de Stevia rebaudiana, en el Medio Sinú. En: Memorias XXXIII Congreso Anual de Comalfi. Montería, Colombia.

Geuns, J. 2003. Stevioside. Phytochem. 64, 913-921.

Geuns, J.; J. Buyse; A. Vankeirsbilck y E. Temme. 2007. Metabolism of stevioside by healthy volunteers. Exp. Biol. Med. 232, 164-173.

Gregersen, S.; P. Jeppesen; J. Holst y K. Hermansen. 2004. Antihyperglycemic effects of stevioside in type 2 diabetic subjects. Metab. Clin. Exp. 53, 73-76.

Guevara-García, A.; C. San Román; A. Arroyo; M. Cortes; M. De la Luz Gutiérrez-Nava y P. León. 2005. Characterization of the arabidopsis clb6 mutant illustrates the importance of posttranscriptional regulation of the methyl-D-erythritol 4-phosphate pathway. Plant Cell 17(2), 628-643. 
Hsieh, M. y H. Goodman. 2005. The arabidopsis IspH homolog is involved in the plastid nonmevalonate pathway of isoprenoid biosynthesis. Plant Physiol. 138(2), 641-653.

Hsieh, M.; P. Chan; Y. Sue; J. Liu; T. Liang; T. Huang; B. Tomlinson; M. Chow; P. Kao y Y. Chen. 2003. Efficacy and tolerability of oral stevioside in patients with mild essential hypertensiona two-year, randomized, placebo-controlled study. Clin. Ther. 25, 2797-2808.

Jarma-Orozco, A. 2005. Cinética del crecimiento. Seminario doctoral. Escuela de Posgrados, Facultad de Agronomía, Universidad Nacional de Colombia, Bogotá

Jarma-Orozco, A. 2006. Adaptación de dos clones de Stevia rebaudiana a tres ambientes del Caribe colombiano. Seminario doctoral. Escuela de Posgrados, Facultad de Agronomía, Universidad Nacional de Colombia, Bogotá.

Jarma-Orozco, A.; R. Montoya y H. Aramendiz. 2003. Stevia rebaudiana, alternativa de sustitución de cultivos ilícitos en el Caribe colombiano. pp. 64-65. En: Memorias XXXIII Congreso de Comalfi. Montería, Colombia.

Jarma-Orozco, A.; T. Rengifo y H. Aramendiz. 2005. Aspectos fisiológicos de estevia (Stevia rebaudiana Bertoni) en el Caribe colombiano. I. Efecto de la radiación incidente sobre el área foliar y la distribución de biomasa. Agron. Colomb. 23(2), 207-216.

Jarma-Orozco, A.; T. Rengifo y H. Aramendiz. 2006. Aspectos fisiológicos de estevia (Stevia rebaudiana Bertoni) en el Caribe colombiano. II. Efecto de la radiación incidente sobre los índices de crecimiento. Agron. Colomb. 24(1), 38-47.

Jeppesen, P., S. Gregersen., K. Alstrupp y K. Hermansen. 2002. Stevioside induces antihyperglycaemic, insulinotropic and glucagonostatic effects in vivo: studies in the diabetic goto-Kakizaki (GK) rats. Phytomed. 9, 9-14.

Jeppesen, P., S. Gregersen; C. Poulsen y K. Hermansen. 2000. Stevioside acts directly on pancreatic a cells to secrete insulin: actions independent of cyclic adenosine monophosphate and adenosine triphosphate-sensitive $\mathrm{K}^{+}$channel activity. Metabolism 49, 208-214.

Jordán, M., F. 1984. Publicación miscelánea No. 11. El $\mathrm{Ka}^{\prime}$ a He'e, Stevia rebaudiana (Bertoni). Análisis bibliográfico y anotaciones hortícolas. Ministerio de Agricultura y Ganadería, Asunción, Paraguay.
Kasahara, H.; A. Hanada; T. Kuzuyama; M. Takagi; Y. Kamiya y S. Yamaguchi. 2002. Contribution of the mevalonate and methylerythritol phosphate pathways to the biosynthesis of gibberellins in arabidopsis. J. Biol. Chem. 277(47), 45188-45194.

Koyama, E.; N. Sakai; Y. Ohri; K., Kitazawa; O. Izawa; K. Kakegawa; A. Fujino y M. Ui. 2003. Absorption and metabolism of glycosidic sweeteners of stevia mixture and their aglycone, steviol, in rats and humans. Food Chem. Toxicol. 41, 875-883.

Lee, C.; K. Wong; J. Liu; Y. Chen; J. Chen y P. Chan. 2001. Inhibitory effect of stevioside on calcium influx to produce antihypertension. Planta Medica 67, 796-799.

Lizaso, J.; W. Batchelor y M. Westgate. 2003. A leaf area model to simulate cultivar-specific expansion and senescence of maize leaves. Field Crops Res. 80, 1-17.

Mansab A.; D.L. Jeffers y P.R. Henderlong. 2003. Interrelationship between leaf area, light interception and growth rate in a soybean-wheat system. Asian J. Plant Sci. 2(8), 605-612.

Marín, W. 2004. Sondeo de mercado de la Estevia. Instituto de Investigación de Recursos Biológicos Alexander von Humboldt, Bogotá.

Marschner, H. 2002. Mineral nutrition of higher plants. Academic Press, New York, NY.

Ministerio de Agricultura y Ganadería del Paraguay. 1996. Producción de Ka’a He’e. 3a. ed., Asunción.

Peng, S.; J. Huang; X. Zhong; J. Yang; G. Wang y Y. Zou. 2002. Research strategy in improving fertilizer nitrogen use efficiency of irrigated rice in China. Sci. Agr. Sinica 35, 1095-1103.

Prakash, I.; G.E. DuBois; J.F. Clos; K.L. Wilkens y L.E. Fosdick. 2008. Development of rebiana, a natural, noncaloric sweetener. Food Chem. Toxicol. 46, S75-S82

Rengifo, T.; A. Jarma y J. Clavijo. 2003. Crecimiento de Stevia rebaudiana bajo cuatro niveles de radiación incidente. p. 66. En: Memorias XXXIII Congreso de Comalfi. Montería, Colombia.

RIRDC, Rural Industries Research and Development Corporation. 2005. A new rural industry -Steviato replace imported chemical sweeteners. Publication No. W02/022. En: http://www.rirdc.gov.au; consulta: agosto de 2006.

Sagakuchi, M. y K. Tatsuiko. 1982. As pesquisas japonesas com Stevia rabaudiana (Bert.) Bertoni e o esteviosideo. Cienc. Cult. 34(2), 235-248. 
Sánchez, D.; K. Huamaní; E. Pascual; H. Sánchez y R. Estrada. 2005. Efecto de las auxinas y citoquininas en la callogénesis sobre explantes nodales e internodales de Tropaeolum tuberosum (r. and p.) Mashua. p. 155. En: Memorias XIV Reunión Científica ICBAR, Lima.

Schwebel, R. 2005. Stevia, el edulcorante natural sudamericano con cero calorías. En: http://www.geocities. com/schwebel_rene/; consulta: junio de 2006.

Secretaría de Agricultura de Antioquia. 2000. Informe preliminar sobre adaptación de la especie Stevia rebaudiana en la región tropical. Gobernación de Antioquia, Medellín, Colombia.

Solange, E., J. Pereira; A. Ramalho; N. Arbex; M. Cardoso y O. Alves. 2002. Indução de calos em erva-detouro (Tridax procumbens L.) utilizando diferentes reguladores de crescimento e tipos de explantes. Cienc. Agrot. 26(2), 301-308.

Suárez, I. y J. Salgado. 2008. Propagación in vitro de Stevia rebaudiana Bert. (Asteraceae-Eupatorieae) a través de organogénesis. Temas Agr. 13(1), 40-48.

Tadhani, M.; V. Patel y S. Rema. 2007. In vitro antioxidant activities of Stevia rebaudiana leaves and callus. J. Food Compos. Anal. 20, 323-329.
Tewolde H.; K. Sistani; D. Rowe; A. Adeli y T. Tsegaye. 2005. Estimating cotton leaf area index nondestructively with a light sensor. Agron. J. 97, 11581163.

Totté, N.; L. Charon; M. Rohmer; F. Compernolle; I. Baboeuf y J. Geuns. 2000. Biosynthesis of the diterpenoid steviol, an entkaurene derivative from Stevia rebaudiana Bertoni, via the methylerythritol phosphate pathway. Tetrahedron Lett. 41, 64076410.

Totté, N.; W. Van den Ende; E. Van Damme; F. Compernolle; I. Baboeuf y J. Geuns. 2003. Cloning and heterologous expression of early genes in gibberellin and steviol biosynthesis via the methylerythritol phosphate pathway in Stevia rebaudiana Bertoni. Can. J. Bot. 81(5), 517-522.

Vergara, C.; A. Jarma; J. Polo y L. Pastrana. 2003. Crecimiento y desarrollo de dos variedades de Stevia rebaudiana en tres tipos de suelo del Medio Sinú. p. 68. En: Memorias XXXIII Congreso Anual de Comalfi. Montería, Colombia.

Yan, X.; P. Wu; H. Ling; G. Xu; F. Xu y O. Zhang. 2006. Plant nutriomics in China: An overview. Ann. Bot. $98,473-482$. 